\title{
STRATEGIC, TACTICAL AND REAL-TIME PLANNING OF LOCOMOTIVES AT NORFOLK SOUTHERN USING APPROXIMATE DYNAMIC PROGRAMMING
}

\author{
Warren B. Powell, Belgacem Bouzaiene-Ayari \\ Department of Operations Research and Financial Engineering, Sherrerd Hall, \\ Princeton University, Princeton, NJ 08544, USA \\ Clark Cheng, Ricardo Fiorillo, and Sourav Das \\ Norfolk Southern Corporation \\ Operations Research \\ 1200 Peachtree St. NE, Box 117, Atlanta, GA 30309, USA \\ Coleman Lawrence \\ Norfolk Southern Corporation \\ Operations and Locomotive Control \\ 1200 Peachtree St. NE, Box 134, Atlanta, GA 30309, USA
}

\begin{abstract}
Locomotive planning has been a popular application of classical optimization models for decades, but with very few success stories. There are a host of complex rules governing how locomotives should be used. In addition, it is necessary to simultaneously manage locomotive inventories by balancing the need for holding power against the need for power at other yards. At the same time, we have to plan the need to return foreign power, and move power to maintenance facilities for scheduled FRA appointments. An additional complication arises as a result of the high level of uncertainty in transit times and delays due to yard processing, and as a result we may have to plan additional inventories in order to move outbound trains on time despite inbound delays. We describe a novel modeling and algorithmic strategy known as approximate dynamic programming, which can also be described as a form of "optimizing simulator" which uses feedback learning to plan locomotive movements in a way that closely mimics how humans plan real-world operations. This strategy can be used for strategic and tactical planning, and can also be adapted to real-time operations. We describe the strategy, and summarize experiences at Norfolk Southern with a strategic planning system.
\end{abstract}

\section{INTRODUCTION}

Locomotive planning is one of the most complex operational problems in freight transportation. Planners have to take into consideration a host of operational characteristics that describe a locomotive to best utilize the fleet to meet the service requirements of the trains. Locomotive fleets can represent billions of dollars in investments, and as a result railroads have every incentive to manage this investment as efficiently as possible. The complexity of the problem has put it well past the capabilities of even today's advanced optimization solvers. Completely overlooked in these models are the important sources of uncertainty such as transit time delays, the dynamics of scheduling commodities such as coal and grain, and the ever present problem of equipment failures and maintenance.

Railroads face three classes of planning problems when managing railroads:

- $\quad$ Strategic planning - The major question here is fleet size and mix, but other questions can include understanding the impact of improvements in transit time reliability, the effect of changes in train plans and changes in interchange policies with other railroads.

- Tactical planning and operational forecasting - Here the horizon is 1-7 days, and the question is whether there will be significant shortages of power that might require additional repositioning, short-term modifications in leasing decisions and perhaps the decision to retain locomotives that belong to other railroads (known as "foreign power") and incur additional per diem charges.

- Real-time planning - The decision here is the assignment of specific locomotives to specific trains that are departing over the immediate horizon (typically the next 12 hours). 
A large railroad may have billions invested in their fleet of locomotives. Too many locomotives mean that hundreds of millions are invested in equipment that is not yielding a return. However, a failure to maintain a sufficiently large fleet translates to delayed trains and service failures that can seriously impact revenue. Despite the scale of this investment, it is not unusual for a large railroad to plan their locomotive fleets using a simple linear regression that relates operating statistics such as forecasted tonnage and operating speeds to the amount of power required to run the railroad.

Given the size of the investment, railroads have tried for decades to tap the power of optimization tools to manage their fleets more effectively. Booler (1980) presents a very early attempt at using linear programming to solve a scheduling model. Chih (1986) and Chih et al. (1990) describe an implementation of an integer programming model for locomotive scheduling at the Burlington Northern Sante Fe Railroad (see also Forbes (1990)). This early work struggled with the limitation of integer programming algorithms, despite using highly simplified models of locomotive operations. A number of advances have been made in the design of specialized algorithms to solve integer programming formulations locomotive scheduling. Ziarati (1999) presents a new branch and cut algorithm. Ahuja et al. (2005) describe a heuristic based on very large scale neighborhoods to find nearoptimal schedules for locomotives which considers consist breakups and the desire for weekly patterns in the flows of locomotives. Vaidyanathan et al. (2008a) provides a detailed model of the locomotive routing problem capturing a number of operational constraints with an adaptation of their large neighborhood search strategy (see also Vaidyanathan et al. (2008b) for additional experimental work). Cordeau et al. (2000, 2001) describe the use of Benders decomposition for the simultaneous assignment of cars and locomotives. At the same time, it is important to recognize major advances to general purpose integer programming solvers such as Cplex and Gurobi that have occurred since 2005. Below, we report on experiments with Cplex 12 running on a multithreaded machine.

This paper describes a multiyear effort to develop a family of locomotive planning models for Norfolk Southern Railroad. The result is the Princeton Locomotive And Shop Management system (PLASMA), which can be used for strategic planning, short term operational forecasting, and real-time operations. PLASMA has been imbedded in a larger information system developed at Norfolk Southern called the Locomotive Assignment and Routing System (LARS). As of this writing, the system has been used for strategic fleet sizing for several years and has become an integral part of the company's network and resource planning processes. The operational forecasting system has been undergoing extensive user acceptance testing while Norfolk Southern has been upgrading its information systems to improve the accuracy of some of the data. We then provide an indication of how the system can be implemented as a real-time, interactive system.

\section{LOCOMOTIVE OPERATIONS}

Locomotives are described by a host of attributes including horsepower and tractive effort, the owning railroad, its maintenance status (e.g. days until the next federally mandated maintenance appointment), and equipment details such as communications gear (for coordinating multiple locomotives). Locomotives typically need to be bundled together into consists of one to perhaps five locomotives which are needed to pull a particular train. The process of connecting locomotives is timeconsuming, requiring the connecting and testing of cables that allow the set of locomotives to work as a single unit. Trains often arrive from a neighboring railroad using locomotives owned by that railroad (known as "foreign power"). Normally foreign locomotives are returned to the owning railroad, but it is possible to use these locomotives, but only within negotiated limits. If the locomotive is returned, this has to be handled through pre-defined exchange points.

The trains themselves also have numerous attributes. The number of locomotives needed to pull a train depends on the weight of the train, the speed requirements (merchandise trains need to move more quickly than coal and grain trains), and the steepest grade that the train has to navigate. Trains have different service priorities, and a scheduler has to consider if there are not enough locomotives to move all the trains on time.

Shop routing (getting the locomotive to a maintenance shop) is one of the most complex issues facing a locomotive manager. Locomotives need to have regular maintenance reviews on a periodic schedule (typically every 90 days). If a locomotive does not make its shop appointment on time, it has to be turned off and "towed" to the shop. Of course, on some trains it is possible to simply add a locomotive as an extra locomotive and move it to its shop appointment, but it is better to use the locomotive productively. At the same time, the scheduler has to balance getting the locomotive to shop early (which costs productivity) or risk that it may arrive late (for example, by missing a critical connection). On top of this, there may be multiple shop locations that can service a locomotive. It is necessary to anticipate the number of locomotives that are scheduled at each shop in order to balance the loads and maintain a steady flow of work.

A separate issue that is widely discussed but rarely solved concerns the different types of uncertainty that plague locomotive operations. These include:

- $\quad$ Transit time delays - These can be as long as six to 12 hours for the shorter movements of an Eastern railroad, to more than a day for the long movements of the Western railroads.

- Dynamic schedule changes - Planners also have to deal with the pattern of scheduling additional trains for commodities such as coal and grain, with as little as one or two days advance notice.

- Shop delays - Maintenance managers will provide estimates of when a locomotive will be ready to leave a shop, but these are just estimates and frequent calls to a maintenance facility are often needed to determine if a locomotive will be ready for a particular train. 
- Equipment failures - Locomotives may fail unexpectedly, and this represents an additional source of uncertainty.

The model presented in this paper is designed to handle uncertainty, but production applications of the model have yet to exploit this capability.

\section{DETERMINISTIC OPTIMIZATION MODELS}

The most common strategy for modeling locomotives as an optimization problem is to use the framework of multicommodity flows over a time-space network, depicted in figure 1.

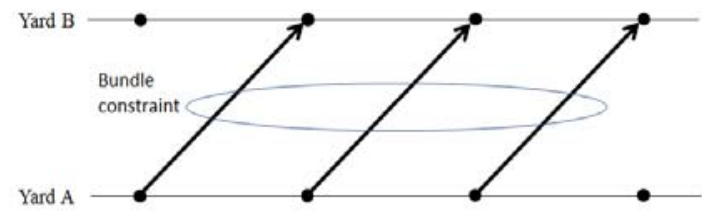

Figure 1-Time-space representation of trains with multiple departure times.

Mathematically, such a model can be written in a generic format using

$$
\min _{x} \sum_{t=1}^{T} \sum_{k=1}^{K} c_{i j}^{k} x_{t i j}^{k}
$$

subject to

$$
\begin{aligned}
& A_{t}^{k} x_{t}^{k}-B_{t-1}^{k} A x_{t-1}^{k}=R_{t} \\
& D_{t} x_{t} \leq u_{t} \\
& x_{t i j}^{k} \geq 0 \text { and integer }
\end{aligned}
$$

Here, $x_{t i j}^{k}$ represents the flow of locomotives of type $k$ moving from yard $i$ to yard $j$ departing at time $t$. Equation (2) captures flow conservation for locomotives of each type. Equation (3) captures the number of locomotives needed to move a train (summing across different types of locomotives). Equation (4) requires that the flow variables be integer.

This basic model has to be adjusted to allow for the following:

- If a train requires three locomotives to meet speed and grade requirements, we can still use the train to pull more locomotives if we need to reposition power from one yard to another.

- One or more locomotives can be moved from one yard to another without pulling a train, an operation known as a "light engine move." Light engine moves incur crew costs and as a result need to be carefully managed.

- There is a cost for coupling locomotives together into a consist to pull a single train, as well as a cost for decoupling the locomotives. This operation also takes time which has to be built into the dynamics.
- Locomotives that need to be routed to shop have to be modeled.

A particularly difficult challenge is the modeling of train delay. The most common assumption is to use a time-space network as shown in figure 1 , but to then replicate train movements over multiple time periods. Since the same train cannot move at different points in time, they are linked by a bundle constraint that ensures that only one "copy" of the train can move, as shown in figure 2 .

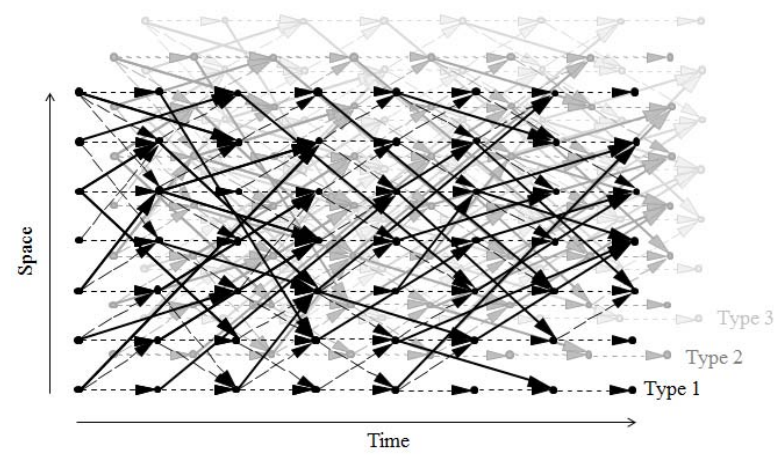

Figure 2- Illustration of multicommodity network flow problem over a time-space network.

Our experience with this strategy was that it introduced discretization errors that were unacceptable to the railroad. If we use a one-hour time step, the network simply explodes. However, if we use a slightly more manageable four-hour time step (which still increases the size of the problem dramatically), we encounter situations where a locomotive may arrive 20 minutes too late to serve a train, but this then forces us to impose a four hour delay. This was felt to be an unacceptable distortion, and it dramatically inflated train delay.

We avoid this problem by using a classic "task graph" formulation, where a train is modeled as a task which is characterized by an initial time of availability. The basic idea is illustrated in figure 3. Locomotives are modeled as arriving in

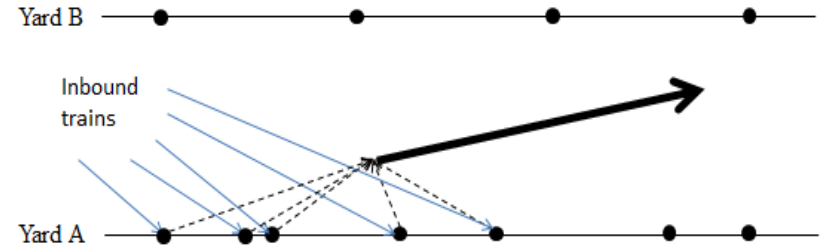

Figure 3-Task graph representation of trains and locomotive arrivals.

continuous time. Dashed assignment arcs can join a locomotive at any point in time to a specific train. The departure time of the train is governed by the latest locomotive that is actually assigned to the train, which allows us to model train delays of any length. 
Deterministic models are extremely hard to solve over long planning horizons, especially when modeling the ability to delay trains. Figure 4 shows the run times as the horizon is extended for the Norfolk Southern fleet. Note the exceptionally fast execution time for the single-day horizon. For this problem, locomotives are being assigned to at most a single train. As the horizon grows, we have to model the cascading of train delays in the task graph. With a horizon of as little as four days, the run times are already exceeding 50 hours.

\section{AN APPROXIMATE DYNAMIC PROGRAMMING MODEL AND ALGORITHM}

Approximate dynamic programming is a modeling and algorithmic strategy that decomposes decisions over time (see Powell (2011) for an introduction using the concepts and notation in this paper; approximate dynamic programming is closely related to the field of reinforcement learning, see Sutton and Barto (1998)). It was originally developed to handle problems which involve uncertainty. Our own work, however, has focused on its ability to decompose large deterministic problems, overcoming the dramatic increase in CPU times documented in figure 4. In fact, all of the production applications of ADP at Norfolk Southern have been conducted using a deterministic model. In this paper, we report on some recent experiments using ADP to improve the robustness of the solution in the presence of uncertainty in transit times.

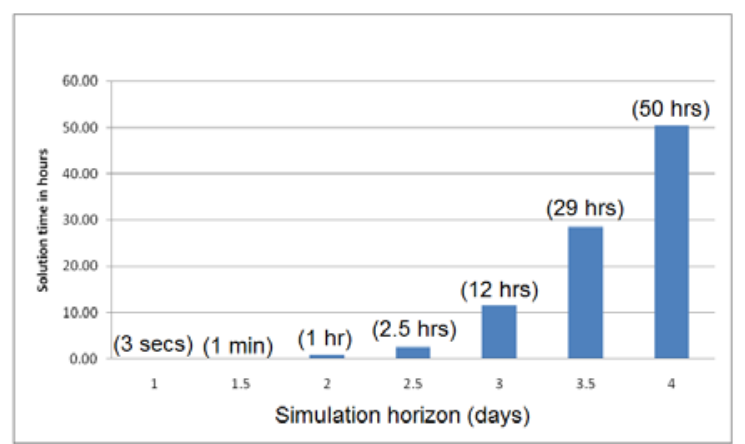

Figure 4 - Execution times for increasing planning horizons for the full fleet of locomotives.

This paper will not attempt to present a complete mathematical model. Instead, we provide a sketch of how approximate dynamic programming approaches the problem. The basic idea in an ADP approach is to set up a subproblem where we assign locomotives to trains over a relatively short horizon (perhaps 4-6 hours). We could start by maximizing the contribution we earn now, ignoring the impact of decisions now on the future. In such a model, let

$S_{t}=$ The state of our system, including the status of current and inbound trains, and the outbound schedule of trains that we know of at time $t$.
$x_{t}=\mathrm{A}$ vector of assignments of current and possibly inbound locomotives to outbound trains over a specific horizon (such as 4-6 hours).

$C\left(S_{t}, x_{t}\right)=$ Contribution earned if we are in state $S_{t}$ and make decision $x_{t}$. This would include bonuses for moving trains (which reflect the priority of the train), costs for forming or breaking consists, and any costs that are incurred implementing the decision.

If we are willing to ignore the future, we would make decisions using a myopic policy defined by $X^{M}\left(S_{t}\right)=$ $\arg \max _{x \in X_{t}} C\left(S_{t}, x_{t}\right)$. This means we find the set of assignments that produces the highest contribution now. Such a policy would never reposition power for use in the future. Furthermore, we might use power now on a low priority train, ignoring the very high priority train that has to leave, say, 10 hours from now. A policy that overcomes this limitation looks like

$$
X^{M}\left(S_{t}\right)=\arg \max _{x \in X_{t}} C\left(S_{t}, x_{t}\right)+\mathrm{E}\left\{V_{t+1}\left(S_{t+1}\right) \mid V_{t}\right\}
$$

where $S_{t+1}=S^{M}\left(S_{t}, x_{t}, W_{t+}\right)$ is the state at the next time period (typically four hours from now) given that we are in state $S_{t}$ right now (this specifies the available locomotives and trains), we make decision $x_{t}$ (this determines which locomotives are assigned to each train, and which locomotives are held), and where $W_{t+1}$ captures the random information such as train delays, schedule changes and equipment problems that were not known at time $t$. In a deterministic implementation, the variable $W_{t+1}$ does not contain anything (no trains are delayed, no schedule changes are made, and no equipment fails). The expectation in equation (5) generally cannot be computed, but we can get around this (for stochastic problems) by using the concept of the post-decision state variable. While this arises in different forms, in this setting it is easiest to think of it as that the state that we would land in if the random information $W_{t+1}$ is what we expect it to be (say, the average travel time, or assuming that there are no changes in the schedule and no equipment failures). Let $\bar{W}_{t+1}$ be a forecast of $W_{t+1}$ given what we know at time $t$. We can write the post decision state $S_{t}^{x}$ using $S_{t}^{x}=S^{M}\left(S_{t}, x_{t}, \bar{W}_{t, t+1}\right)$. We can then replace equation (5) with

$$
X^{V}\left(S_{t}\right)=\arg \max _{x \in X_{t}} C\left(S_{t}, x_{t}\right)+V_{t}^{x}\left(S_{t}^{x}\right) .
$$

Finally, we will never be able to calculate the value function $V_{t}^{x}\left(S_{t}^{x}\right)$ exactly, so we replace it with an approximation that we write as $\bar{V}_{t}^{x}\left(S_{t}^{x}\right) \approx V_{t}^{x}\left(S_{t}^{x}\right)$. The challenge then is to design an approximation $\bar{V}_{t}^{x}\left(S_{t}^{x}\right)$ that is easy to estimate, allows equation (6) to be solved fairly easily, and works well. Recall that the state variable consists of locomotives and trains. We have found that an approximation strategy that works well ignores the value of trains in the future, 
and uses a piecewise linear function to approximate the value of the major classes of locomotive (low/high adhesion, low/high horsepower). Capturing the value of trains in the future contributes to better decisions only when trains are delayed, which happens fairly infrequently. This produces an optimization problem that is depicted in figure 5. In the figure, we see the initial assignment of locomotives to trains. We also use a piecewise linear function (identified as the "train reward function") to capture the value of putting power on the train. Each train has a critical point, below which the train cannot move. We then model the fact that there may be value in adding power above the critical point, but only up to a specified goal horsepower. We can continue to add power because it is needed at a downstream yard, but there is a cost to doing so (the positive contribution is reduced). Finally, we model the value of each type of locomotive at the destination location using piecewise linear value functions. This is done at two levels of aggregation: the value of each of the major types of locomotive at a location, and the value of the total amount of power at a yard. This helps the model learn both the right mix of power, as well as the right amount of power in aggregate.

The policy in equation (6) (and depicted in figure 5) is an

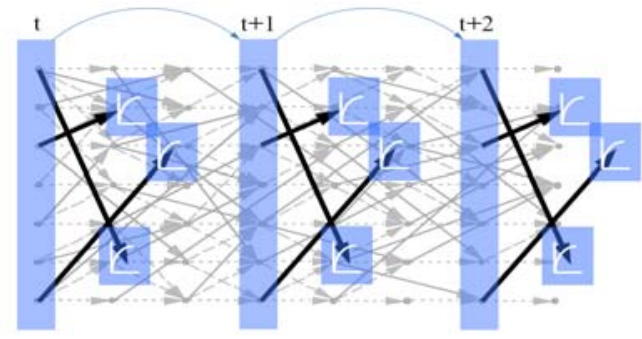

Figure 5-Illustration of the process of stepping forward through time, solving sequences of small locomotive assignment problems with value function approximations.

integer program, but with a very small horizon. As a result, it can be solved in a few seconds. The value function approximations will not be known in advance. These are learned adaptively by computing the marginal value of each type of locomotive at each yard, at each time period. There is by now a fairly extensive literature on these methods, and we

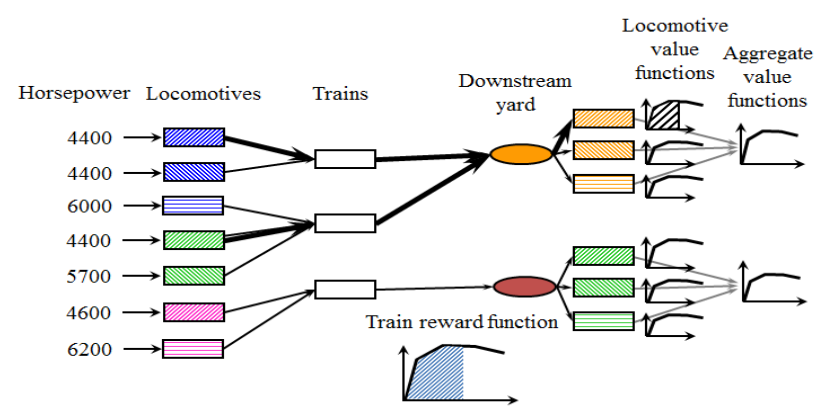

Figure 6-Illustration of the single period decision problem using value function approximations for locomotives in the future. refer the determined reader to Powell (2011) (chapter 13). The basic idea, then, is to start with a value function $\bar{V}_{t}^{n-1}(S)$ which determines our policy (basically, the decision function in equation (6)). We step through time simulating the policy, as depicted in figure 7 , and use the information to produce an

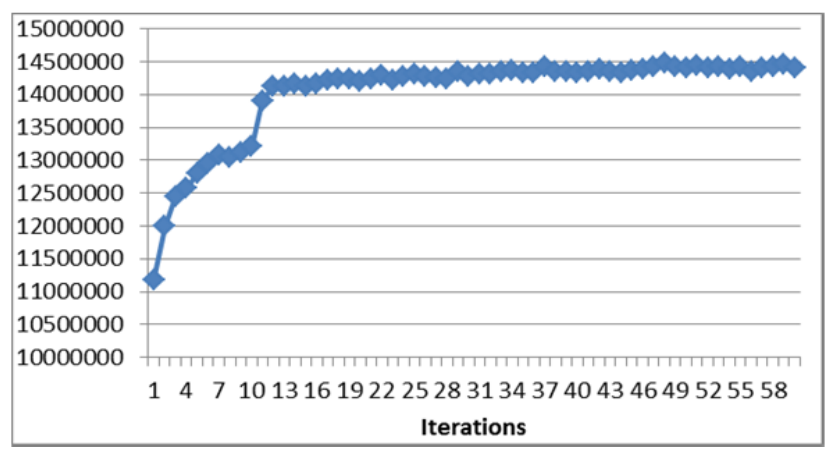

Figure 7-Growth in objective function due to iterative learning

updated value function approximation $\bar{V}_{t}^{n}(S)$. If we want to capture uncertainty, we use Monte Carlo simulation to sample train delays, schedule changes and equipment failures in the information variable $W_{1}, W_{2}, \cdots, W_{t}, \cdots$. However, if we are using a deterministic model, we just simulate the average transit time, and ignore schedule changes and equipment failures. This way, we can solve large deterministic problems using a sequence of very small integer programming problems. The price is that we have to run these simulations iteratively to learn the value function.

The improvement in the objective function as a result of the adaptive learning is shown in figure 7 . Note that the jump in the objective function after 10 iterations is due to an algorithmic strategy where no trains are allowed to be delayed for the first 10 iterations, a strategy that helps to stabilize the value functions. We found that 50 iterations gave good solutions.

A major feature of this strategy, completely separate from the ability to handle uncertainty, is that we can model individual locomotives and trains at a very high level of detail. If we try to simultaneously optimize the problem over a long horizon using a single deterministic formulation, it is essential that the problem be simplified, such as grouping locomotives into a small number of classes (known as "commodities") and discretizing time fairly coarsely. Our strategy makes it possible to handle the different attributes that are required for a truly realistic model. For example, we can capture that a particular locomotive needs to get to shop. During the simulations where we sweep forward in time, we can calculate the time that would be required to get to each shop location, and use this when we solve the problem in equation (5).

This adaptive learning strategy can be used for strategic, tactical and real-time planning. The biggest challenge, however, was calibrating the model so that we could be confident that it was accurately capturing real-world operations. 


\section{MODEL CALIBRATION AND VALIDATION}

The model went through several years of careful calibration against historical performance. This required the painstaking examination of detailed assignments, along with the comparison of high level performance metrics. The process involved the iterative identification and correction of data errors, as well as enhancements in the model and, from time to time, improvements in the basic algorithm. For example, it was through this process that we identified the need to use two layers of aggregation in the value function approximations as shown in figure 5.The most common data problems arose in the initial location of locomotives, and the representation of the train schedule and tonnage requirements. Examples of modeling problems included changes required in the handling of foreign power and the rules for consist formation.

In addition to the careful examination of individual assignments, Norfolk Southern focused on train delay as the most important metric of overall performance. Matching train delay at a system level is an extremely difficult target because it requires that the model match locomotive productivity almost perfectly. For example, it is important that we accurately capture the costs and time required for breaking up locomotive

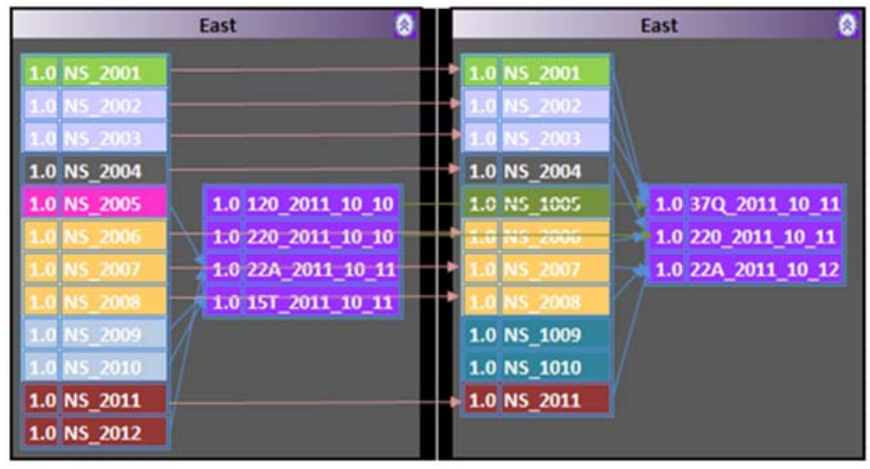

Figure 8 - Snapshot of Pilotview, showing assignments of individual locomotives to trains.

consists. If we ignore this component, we would over-represent the ability to use power to move trains, which in turn would underestimate train delay.

In the early stages of the calibration process, the model would produce delays that were an order of magnitude larger than history, largely as a result of data errors that had locomotives hopelessly out of position. It is not possible to match historical performance simply by tuning parameters within the model. It was essential that the detailed assignments pass the examination of experienced schedulers. This process was simplified by a powerful diagnostic tool called Pilotview (figure 8) that we developed for complex resource allocation problems such as this.

We proceeded by creating a curve from estimates of total train delay as a function of the fleet size. After finally getting the model to closely match historical performance, we repeated the exercise with an entirely new dataset. The result is the curve shown in figure 9, which shows a very close match between the curve and the historical delay at the current effective fleet size. Also note that the relationship between fleet size and train delay is smooth and predictable. Achieving this behavior with a model that captures this level of detail is actually quite hard, as it requires that we have the ability to model train delays continuously.

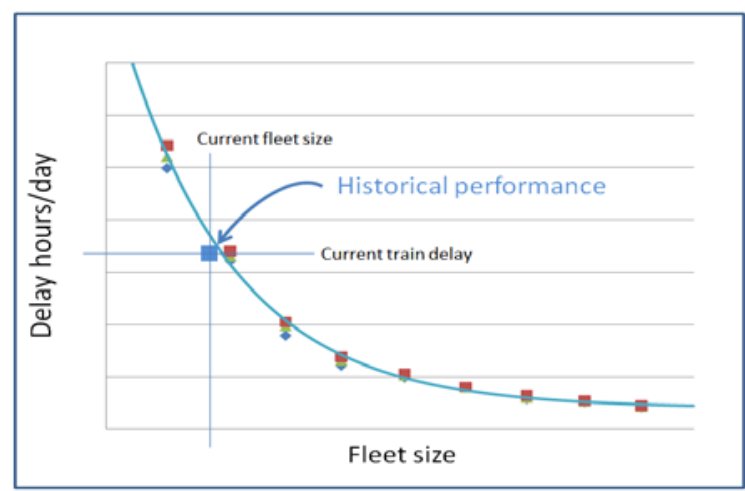

Figure 9 - Simulated train delay versus fleet size, compared to historical performance.

Other forms of model validation involved testing the sensitivity of the model to key input parameters. One such test evaluated the effect of increasing the consist breakup cost to determine its impact on both the number of consists being broken and overall solution quality. Figure 10 shows the effect of increasing the consist breakup cost, using the rate of consist breakups with a cost of zero as a baseline. The chart demonstrates that increasing the consist breakup cost produces a steady decline in the number of broken consists. We note that this was achieved with no discernible reduction in the model objective function which captures repositioning costs and

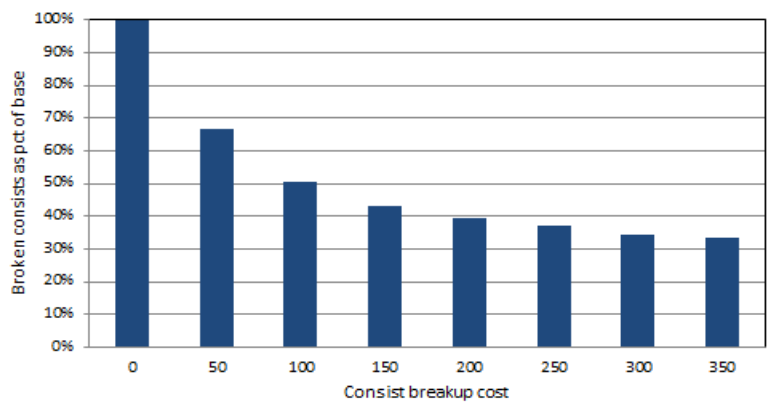

Figure 10 - Effect of the consist breakup penalty on the number of broken consists relative to a base case.

penalties for delayed trains.

The model also has the ability to balance loads across shop locations when routing power to shop. Shop routing is a particularly sophisticated feature of the model. It uses adaptive learning to estimate the time required to get a locomotive to each shop (given all downstream events), and to estimate the backlog at each shop. We can then introduce a penalty to reduce these backlogs. Figure 11 shows the total backlog across all the shop locations as a function of the time within the 
simulation. The model can do little to reduce backlogs early in the simulation, which are largely a result of initial conditions, but with a higher penalty, the backlogs are reduced as the simulation progresses (note that this behavior is learned over the course of about 50 iterations).

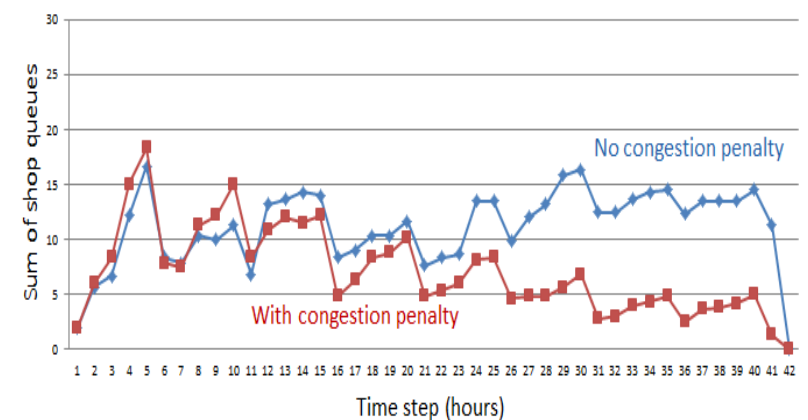

Figure 11-Total shop backlog over the course of a simulation, with and without a congestion penalty.

These features make it possible to tune the model so that it can achieve realistic behaviors. For example, it is important that the model handle consist breakups and shop routing in a realistic way if it is going to be used for fleet sizing. Ignoring these important operational issues would allow the model to achieve levels of utilization higher than what could be achieved in the field, a common problem with the use of optimization models. In addition, these features mean that it is possible to perform strategic planning studies that are realistic to railroad operations.

At this point, we concluded that the model was calibrated, and responded in a smooth and consistent way to changes in the input parameters.

\section{STRATEGIC PLANNING}

The most important strategic planning question at Norfolk Southern involved estimating the appropriate fleet size and mix given a projected train schedule. NS had used a simple regression model to estimate fleet size, but management came to feel that inefficiencies were baked into this model. The development of PLASMA was motivated by the desire to have an engineering solution that could adapt in a realistic way to assumptions about train schedules, fleet size and mix, and network performance.

When the model is used for strategic planning, all locomotives start in a "super source" node. We do not have to specify where locomotives are initially, and we do not even have to specify the fleet mix, although we are allowed to do so. The model then figures out where to first position each locomotive at the beginning of the planning horizon. After this decision is made, the adaptive learning logic assigns power to trains over a planning period (typically a month). The use of an optimization-based modeling strategy means that the model simulates a well-trained group of locomotive planners.
Figure 12 illustrates how the model is used to estimate fleet size. The model is first used to create a train delay curve (total delay as a function of fleet size) for the current year. Then, a projected train schedule is created for some period in the future, after which the model is used to create a new delay curve. If we would like to maintain the same level of train delay, we can simply pick off the required number of locomotives. If we do not constrain the model to a fixed proportion of different locomotive types, the model will also specify the fleet mix.

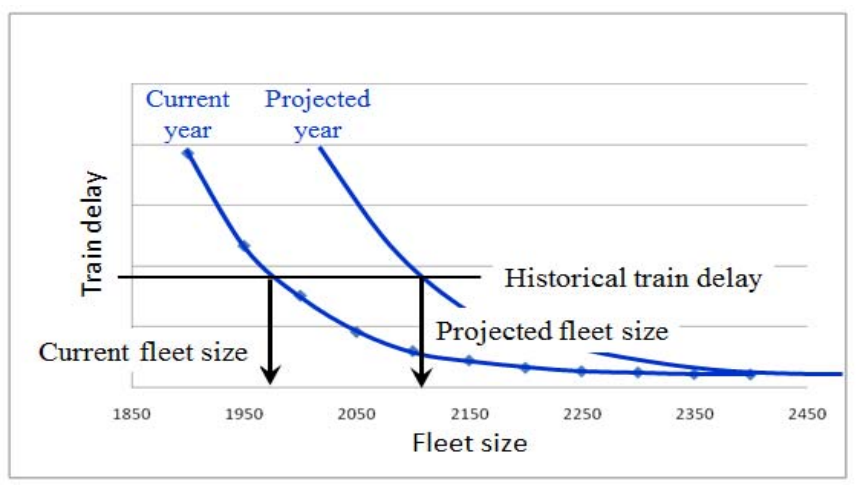

Figure 12 - Delay curves based on current and forecasted schedules, showing the fleet size needed to maintain the same level of train delay.

The model can also be used to perform different types of policy studies. Figure 13 illustrates an analysis of the effect of changes in average train speed. Train delay curves were generated for a base case, and then for six scenarios where the average train speed was varied. We note that the curves are quite consistent and well-behaved, simplifying the task of identifying the correct fleet size.

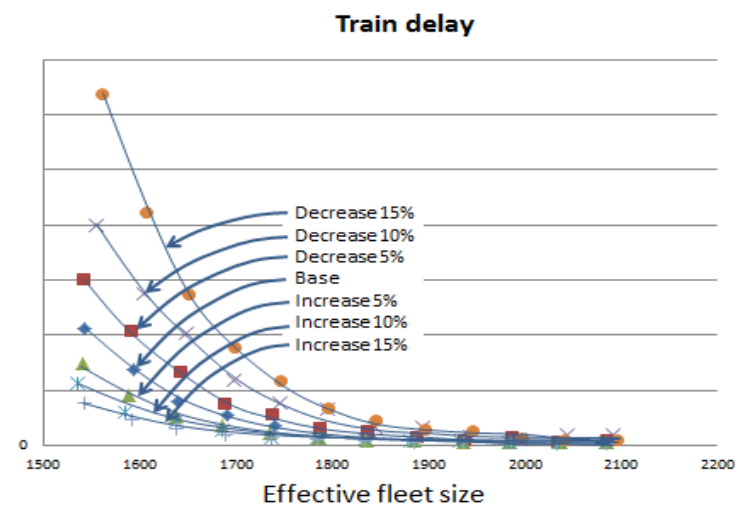

Figure 13- Train delay curves for different variations of average train speed.

While Norfolk Southern has primarily used the model for fleet sizing studies, it can be used for other questions such as quantifying the effect of changes to the train schedule, changes in interchange points to foreign railroads, and changes in the size and location of maintenance shops. 


\section{EXTENSIONS}

We close with a discussion of several significant ways in which the model can be extended. These include: managing uncertainty, using the model for short term operational forecasting, and finally, using the model for real-time operational planning.

\section{MANAGING UNCERTAINTY}

Locomotives have to be managed in the presence of a significant level of uncertainty. The most important is the variability in transit times due primarily to the need for lower priority trains to sit on sidings to allow higher priority trains to pass. Other sources of uncertainty arise as a result of yard delays, changes in the train schedule (e.g. scheduling new coal and grain trains), and equipment problems.

Approximate dynamic programming makes it extremely easy to handle uncertainty. The algorithm requires that we step forward through time repeatedly as we learn the value of locomotives in the future. If we want to incorporate uncertainty, all we have to do is to sample from distributions describing transit times, yard delays and equipment failures. The effect can be quickly seen in the value functions. Figure

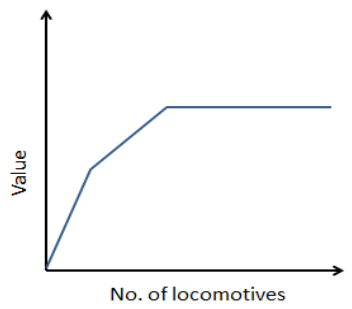

Figure 14(a) - Value function trained on deterministic data.

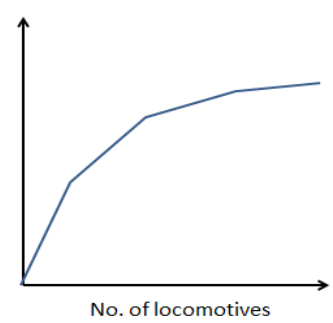

Figure 14(b) - Value

function trained on stochastic data. 14(a) illustrates what a value function might look like if trained on deterministic data. The value rises as a function of the number of locomotives, but there is clearly a point where additional locomotives are just not needed. Figure 14(b) illustrates what the same value function might look like if trained in the presence of uncertainty. Here, the value function continues to rise, because situations might arise where an inbound train is delayed, and as a result there is value in holding additional locomotives.

When we use stochastically trained value functions, there is value to holding additional power in a yard. This reflects the tendency of terminal managers to hold onto a few extra locomotives, just in case of problems. Of course, every yard manager would like to hold onto a few more locomotives, creating the widely recognized problem where the railroad appears to need more locomotives than it has. The logic will create the highest values (in the form of higher slopes) at the yards which truly need the additional power the most. At the same time, just because there is value to holding more locomotives does not mean that a yard will be allowed to keep additional locomotives. Instead of simplistic rules to hold a particular number of locomotives at specific yards, the value functions provide a flexible policy that adapts to the general availability of locomotives. For example, each railroad experiences a time every week when power inventories are at their tightest. This is the point where yards simply cannot hold onto buffer inventories. By contrast, at other times there are yards which really benefit from holding onto power to protect against inbound delays or equipment failures.

Figure 15 shows train delay over 150 simulations of random transit times, using deterministically and stochastically trained value functions. Note that the stochastically trained value functions not only produce smaller delays, but also much

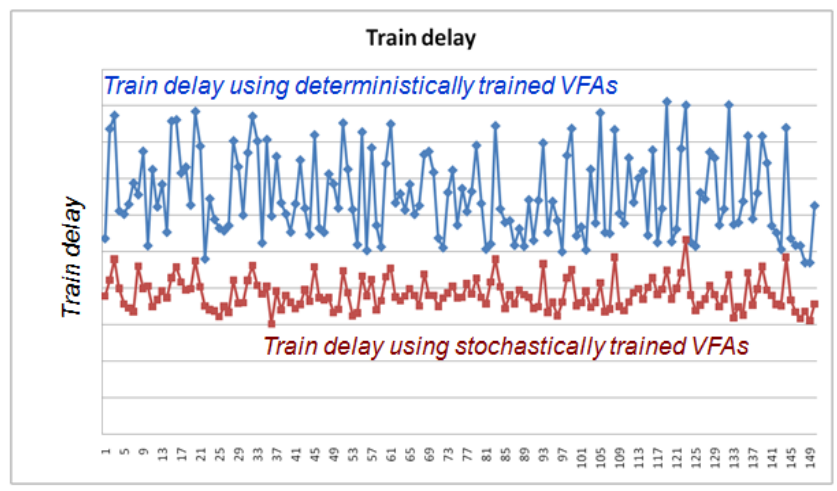

Figure 15 - Train delay over multiple simulations using deterministically and stochastically trained value function approximations.

more stable results. Given the effect of randomness on the value functions (depicted in figure 14), we would expect that the stochastically trained value functions should be more inclined to hold power in inventory. Figure 16 shows that this hypothesis is accurate. When aggregated across the railroad, the stochastically trained VFAs show consistently higher inventories, although the difference varies by time reflecting, we believe, the changing ability of the network to hold power.

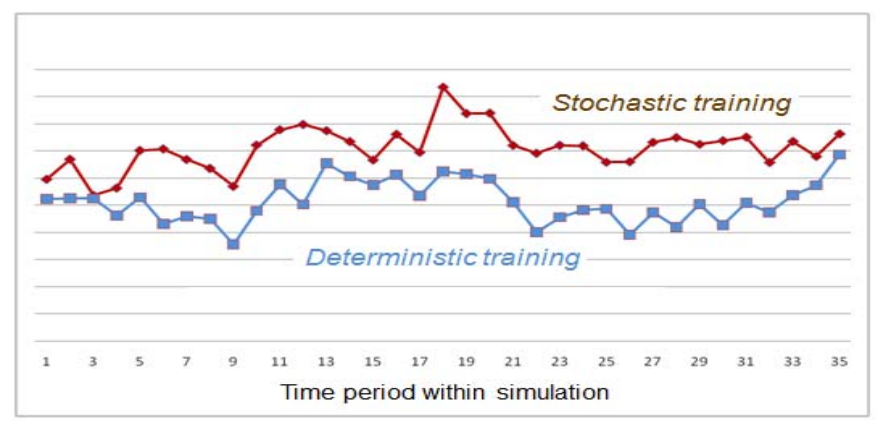

Figure 16-Aggregate power inventories over the course of a simulation using stochastically and deterministically trained value function approximations.

This is the first reported solution of a stochastic formulation of the locomotive management problem. The 
methods presented are based on a mathematically rigorous formulation, but they are also quite intuitive and practical.

\section{OPERATIONAL FORECASTING}

An operational forecasting model produces a plan over perhaps a five to seven day horizon. The model is used to identify surpluses and deficits of power, and to anticipate locomotive repositioning and light engine moves (moving power without a train). Such a model requires that we know where the locomotives are initially. Thus, while the strategic planning model has to figure out where each locomotive should be at the beginning of the simulation, the operational forecasting model works from a live snapshot.

The operational forecasting model at NS runs in a production setting. After each forward sweep (over, say, a seven day horizon), the model would refresh the locomotive snapshot, as well as capture any changes to the train schedule. This process should repeat itself approximately once each minute (for a network comparable to that of Norfolk Southern). In the process, the model is constantly refining the value function approximations.

The operational forecasting model requires that the train schedule and locomotive snapshot be accurate (the strategic planning model does not require a locomotive snapshot). This is not a small request for a railroad. Norfolk Southern has been extensively testing and validating the operational forecasting model, but in the meantime the process has helped to identify areas where data reporting needs to be more accurate. This will be realized through upgrades to the information systems and improved reporting procedures. A byproduct of this implementation has sparked a major revision of their data collection and reporting process for locomotives. This is a familiar experience, where the process of implementing advanced decision support systems has the effect of raising the bar on the quality of information systems.

\section{REAL-TIME PLANNING}

The last and arguably most ambitious use of optimization would be the real-time assignment of locomotives to trains. Real-time assignment models have been used for years for truckload trucking with tremendous success, and several railroads use real-time optimization to assign cars to orders.

PLASMA can easily be adapted to perform real-time operational planning for locomotives. Assuming that the operational forecasting model is running in production, we have access to the value function approximations. A real-time operational model requires that instead of solving a sequence of decision problems (depicted in figure 5) over time (as illustrated in figure 6), we only have to solve a single problem, reflecting what is known now. Such a model can be solved from scratch in a matter of seconds, but it can be solved even more quickly by holding the solution live in memory.

The challenge of any real-time model is that human planners always have access to information that will simply not be available in the computer. For example, often the first source of updated information about the status of a locomotive comes from an inspector talking on a cell phone to a planner. As a result, regardless of the sophistication of a model or the quality of a database, there will always be instances when a human will simply disagree (and in some cases, correctly) with the recommendation of a model. This is not a problem if the planner is allowed to override the model, and if the model can then be updated extremely quickly (which is to say in a second or two). The speed with which the model needs to be updated is not related to the rate at which updates come in from the railroad. The main constraint is the speed with which planners make decisions.

Real-time operational model pose additional demands on the quality of data, over and above what is required for a tactical model. However, we believe that a fully interactive model can be robust, adding value even in the presence of imperfect information.

\section{CONCLUSIONS}

Approximate dynamic programming offers a novel modeling and algorithmic strategy that combines the realism of simulation with the intelligence of optimization. Classical optimization models have offered the promise of better decisions, but the technology has required the use of major simplifying assumptions. As a result, the "savings" produced by such models are often a by-product of simplified models rather than intelligent decisions.

PLASMA has been shown to produce high quality, accurate solutions to strategic and tactical planning problems at Norfolk Southern. Furthermore, it has shown very promising results for operational forecasting and has high potential for real-time locomotive assignments. It is the first optimizationbased model that calibrates accurately against history, making it useful as a tool for fleet sizing, one of the most demanding strategic planning problems. The technology allows locomotives and trains to be modeled at an extremely high level of detail. Train delays can be modeled down to the minute. The model can simultaneously handle consist breakups and shop routing, while also planning the empty repositioning of power. In addition, it can handle uncertainties in transit times, yard delays and equipment failures in a simple and intuitive way. The entire methodology is based on first principles, and as a result avoids the need for heuristic rules that have to be retuned as the data changes.

\section{ACKNOWLEDGMENTS}

We would like to thank the following people for their contributions to this project: Ajith Wijeratne (who was the first to use the train delay curves), Junxia Chang, Don Graab, Wayne Mason, Ed Courtney, Brian Wilker, Jermaine Wilkinson, and Ricky Sansbury. The first author would like to thank the very early support of Roger Baugher who recognized the potential of approximate dynamic programming for rail operations. The 
research behind this work has been supported over the years by the Air Force Office of Scientific Research and the National Science Foundation.

\section{REFERENCES}

[1] Ahuja, R. K., Liu, J., Orlin, J. B., Sharma, D., \& Shughart, L. A. (2005). Solving real-life locomotive-scheduling problems. Transportation Science, 39, 503-517.

[2] Booler, J. (1980). The solution of a railway locomotive scheduling problem. Journal of the Operational Research Society, 31(10), 943-948. Pergamon Press. Retrieved from http://www.jstor.org/stable/2581584

[3] Chih, K., Hornung, M., Rothenberg, M., \& Kornhauser, A. (1990). Implementation of a real time locomotive distribution system. Computer Applications in Railway Planning and Management (pp. 39-49). Computational Mechanics Publications, Southampton, UK.

[4] Chih, K. C.-K. (1986). A Real Time Dynamic Optimal Freight Car Management Simulation Model of the Multiple Railroad, Multicommodity Temporal Spatial Network Flow Problem. Princeton University.

[5] Cordeau, J. F., Soumis, F., \& Desrosiers, J. (2000).A Benders decomposition approach for the locomotive and car assignment problem. Transportation Science, 34, 133149.

[6] Cordeau, J. F., Soumis, F., \& Desrosiers, J. (2001). Simultaneous assignment of locomotives and cars to passenger trains. Operations Research, 49, 531-548.
[7] Forbes, M., Holt, J., \& Watts, A. (1991).Exact solution of locomotive scheduling problems. Journal of the Operational Research Society, 825-831.

[8] Powell, W.B. (2011) Approximate Dynamic Programming: Solving the curses of dimensionality, 2nd edition, John Wiley and Sons, Hoboken, NJ.

[9] Rouillon, S., Desaulniers, G., \& Soumis, F. (2006).An extended branch-and-bound method for locomotive assignment. Transportation Research Part B: Methodological, 40(5), 404-423. doi:10.1016/j.trb.2005.05.005

[10] Sutton, R. and A. Barto, (1998) Reinforcement Learning, MIT Press, Cambridge, MA.

[11] Vaidyanathan, B., Ahuja, R. K., \& Orlin, J. B. (2008a). The Locomotive Routing Problem. Transportation Science, 42(4), 492-507. doi:10.1287/trsc.1080.0244

[12] Vaidyanathan, B., \& Ahuja, R. K. (2008b). Real-life locomotive planning: New formulations and computational results. Transportation Research B, 42, 147-168

[13]Ziarati, K., Soumis, F., Desrosiers, J., Gelinas, S., \&Saintonge, A. (1997). Locomotive assignment with heterogeneous consists at CN North America. European journal of operational research, 97, 281-292.Elsevier.

[14]Ziarati, K., Soumis, F., Desrosiers, J., \& Solomon, M. (1999).A branch-first, cut-second approach for locomotive assignment. Management Science, 45, 1156-1168. 